\title{
IX. On two pulsating spheres in a liquid
}

\section{A. L. Selby M.A.}

To cite this article: A. L. Selby M.A. (1890) IX. On two pulsating spheres in a liquid, Philosophical Magazine Series 5, 29:176, 113-123, DOI: $10.1080 / 14786449008619909$

To link to this article: http://dx.doi.org/10.1080/14786449008619909

$$
\text { 册 Published online: } 14 \text { Oct } 2011 .
$$

Submit your article to this journal

Џ Article views: 2

Q View related articles ¿ 
series $m$ is a whole number, whereas in the odd series it is a whole number and a half, thus corresponding to the wellknown difference between elements of even series on the one hand and odd series on the other.

Again, in the arithmetical series,

$$
0 ; 2 \frac{1}{2} ; 5 ; 8 \frac{1}{2} ; 12 ; 15 \frac{1}{2} ; 19 ; 22 \frac{1}{2} ; 26 ; 29 \frac{1}{2} ; 33
$$

the common difference in the case of the first three members is only $2 \frac{1}{2}$, whereas after that point it is $3 \frac{1}{2}$; this corresponds to the statement of Mendeljeff that the 2 nd and 3rd series (i.e. Ii and $\mathrm{Na}$ series) of elements are more or less exceptional in their character and not strictly comparable with the subsequent series.

IX. On two Pulsating Spheres in a Liquid. By A. L. Skidi, M.A., Fellow of Merton College, Oxford*

A SPHERE is said to pulsate when it periodically dilates

If two pulsating spheres are immersed in a liquid, inequalities of pressure in the liquid arise, and there is generally a resultant pressure on each sphere urging it towards or away from the other.

An approximate expression of this is given in Basset's 'Hydrodynamics' (vol.i. p. 255), but the proof is rather long, involving an integration of pressures over the surface of a sphere.

By a somewhat different method I have obtained in $\$ 5$ an expression which may be carried further than Mr. Basset's without much labour; and in $\$ 6 \mathrm{I}$ have applied the method to solve the more general problem relating to the case when the radial velocities on the surfaces of the spheres are Zonal Harmonics of the $m$ th and $n$th degrees respectively, with the line of centres as their axis.

A still more general solution can be derived from this ; for when the radial velocities on the spheres are any functions of the distance from the line of centres, each can be expressed as a series of Zonal Harmonics.

Since I wrote this paper I have found one by Prof. Pearson (Trans. Camb. Phil. Soc. vol. xiv. part ii.) in which this problem is investigated; but numerical values of the constants involved are only given for Harmonics of low degree.

In dealing with these questions some propositions on

* Communicated by the Author.

Phil. Mag. S. 5. Vol. 29. No. 176. Jan. 1890. 
Images, due to Mr. Hicks, are required. His proofs will be found in the 'Philosophical Transactions' for 1880; but in $\S \S 2-4$ of this paper I give other proofs, in which the subject is considered from a slightly different point of view.

The main interest of the problem of pulsating spheres is derived from its experimental illustration by Prof. Bjerknes.

$\$ 1$. Preliminary Definitions. - A source of liquid of strength $m$ is a point at which a volume of liquid $4 \pi m$ is supplied per: unit time. A sink (or negative source) of equal strength is a point at which liquid disappears at the same rate. A combination of a source and sink of equal numerical strength $m$, at an infinitesimal distance $d$ apart, is a doublet. As this is analogous to an infinitely small magnet, I shall call $m d$ the moment of the doublet. The intensity of a doublet is its moment divided by its length; it is analogous to the intensity of magnetization of a bar of unit section. The axis of a doublet is the line from the sink to the source.

The lines of flow due to any distribution of sources and sinks are the lines of force due to the corresponding magnetic poles. line OL:

$$
\mathrm{TPL}=\theta ; \quad \mathrm{TP}^{\prime} \mathrm{L}=\theta^{\prime} ; \quad \mathrm{TP}=r ; \quad \mathrm{PP}^{\prime}=d .
$$

Then the flow through the circle (of which $\mathrm{TT}^{\prime}$ is the projection) formed by revolving $\mathrm{T}$ round $\mathrm{OL}$ is

$$
2 \pi m\left(\cos \theta^{\prime}-\cos \theta\right) \text {. }
$$

Fig. 1.

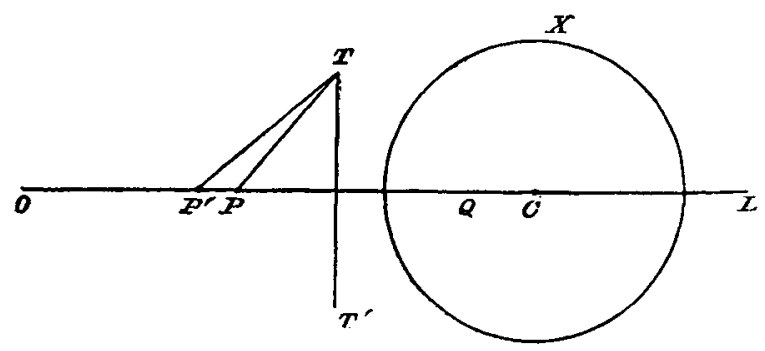

Let $\mathrm{P}^{\prime}$ approach $\mathrm{P}$ so that $\mathrm{PP}^{\prime}$ diminishes indefinitely, $m d$ retaining a constant magnitude $\mu$. Then

$$
2 \pi m\left(\cos \theta^{\prime}-\cos \theta\right)=2 \pi \mu \sin ^{2} \theta \cdot / r \text {. }
$$

If the source and sink are interchanged, the sign of the flow is reversed; we may consider $\mu$ as negative in this case. 
Let a doublet of moment $-\mu^{\prime}$ be placed at $\mathrm{Q}$, where $\mu>\mu^{\prime}$. If $\mathrm{TQL}=\phi, \mathrm{TQ}=r^{\prime}$, the flow through $\mathrm{TT}^{\prime}$ due to this is

$$
-2 \pi \mu^{\prime} \sin ^{2} \phi \cdot / r^{\prime}
$$

The equation of a surface ot revolution across which there is no flow from the doublets at $\mathrm{P}$ and $\mathrm{Q}$ is

$$
2 \pi\left(\mu \sin ^{2} \theta \cdot / r-\mu^{\prime} \sin ^{2} \phi \cdot / r\right)=\mathrm{A}, \text { a constant. }
$$

If $A=0$, this reduces to the line $\mathrm{OL}$ and the sphere $\mu r^{-3}=\mu^{\prime} \gamma^{j-3}$.

If $\mathrm{C}$ be the centre of this sphere and $a$ the radius,

$$
\mathrm{CP} . \mathrm{CQ}=a^{2} \text { and } a=\left(\alpha^{2}-1\right) \mathrm{PQ} / 2 \alpha \text {, }
$$

where $\alpha^{3}=\mu / \mu^{\prime}$.

Since the liquid is supposed to be "perfect," and the sphere is part of a surface of flow, we may make a material sphere occupy its place.

Hence the effect of a sphere $\mathrm{X}$ (fig. 1) in disturbing the flow from the doublet $\mu$ at $\mathrm{P}$, distant $f$ from its centre, is the same as if the sphere were removed and a doublet $-\mu a^{3} / f^{3}$ placed at Q. This doublet is called the image of $\mu$ in the sphere.

\$3. To determine the inage of a source $\mu$ at $\mathrm{P}$.

This source is equivalent to a line of doublets of intensity $\mu$ extending from $\mathrm{P}$ to infinity along $\mathrm{PO}$ (fig. 1). Its image is therefore a line of doublets from $Q$ to $\mathrm{C}$.

If $\mu d$ be the moment of a doublet distant $a^{2} / x$ from the centre, $-\mu d x^{3} / a^{3}$ is the moment of its image. But the lengths of corresponding parts of $\mathrm{P} \infty$ and $\mathrm{CQ}$ are as $a^{2}: x^{2}$.

Therefore the intensity of the image is $-\frac{\mu x}{a}$, supposing the axis along $\mathrm{QC}$; or $\frac{\mu x}{a}$, regarding it as along $\mathrm{CQ}$.

Now a magnet of length $l$ and unit section, whose intensity of magnetization at a distance $x$ from one end is $\mathrm{C} x$, is equivalent (so far as concerns external action) to a quantity of "magnetic matter" $\mathrm{Cl}$ at the positive end, and a distribution of line-density $-\mathrm{C}$ along the axis.

Therefore, from the analogy between sources and magnetic poles, the line of doublets is equivalent to a source $\mu a / f$ at $Q$, and a line sink of strength $-\mu / a$ per unit length along $\mathrm{CQ}$.

$\S 4$. To find the image of a system of doublels $\mathrm{PQ}$ (fig. 2) of intensity $\mu \mathrm{x}$, where $\mathrm{x}$ is the distance from $\mathrm{Q}$.

Let $\mathrm{P}^{\prime}, \mathrm{Q}^{\prime}$ be the points inverse to $\mathrm{P}$ and $\mathrm{Q}$.

By $\S 2$ the image is a system of doublets lying between $\mathrm{P}^{\prime}$ and $\mathrm{Q}^{\prime}$. 
Let $\mathrm{N}$ be a point on $\mathrm{PQ}, \mathrm{N}^{\prime}$ its image, $\mathrm{QN}=x, \mathrm{CP}=f$.

By $\S 3$ the image of a doublet of intensity $\mu x$ at $\mathrm{N}$ is of intensity $-\mu x \mathrm{CN}^{\prime} / a$ or $-\mu \mathrm{CQ} \cdot \mathrm{Q}^{\prime} \mathrm{N}^{\prime} / a$.

Fig. 2.

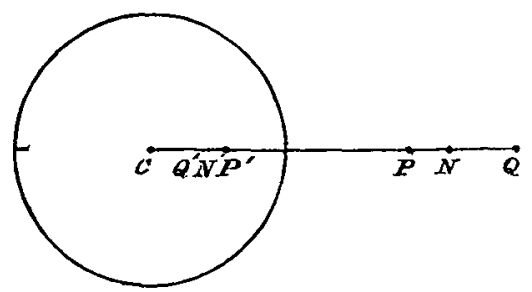

The image of the system $\mathrm{PQ}$ is therefore a line of doublets in which the intensity is proportional to the distance from $Q^{\prime}$, and the image is similar to the system from which it is derived.

This result is easily transformed by the process used in $\S 3$ into the following :-

The image of a source $\mu$ at $P$ and a line-sink $P Q$ of strength $-\frac{\mu}{\mathrm{PQ}}$ per unit length is a source $\mu . a / f$ at $\mathrm{P}^{\prime}$ and a line-sink $\mathrm{P}^{\prime} \mathrm{Q}^{\prime}$ of strength $-\frac{\mu a}{f \cdot \overline{\mathrm{P}}^{\prime} \mathrm{Q}^{\prime}}$

It is clear that any system of sources and sinks of total strength zero distributed along $P Q$ has for its image another such system distributed along $P^{\prime} Q^{\prime}$. For the given system can be reduced to a system of doublets.

§5. To determine the stress between two spheres $\mathrm{X}, \mathrm{Y}$, pulsating in an infinite liquid.

Fig. 3.

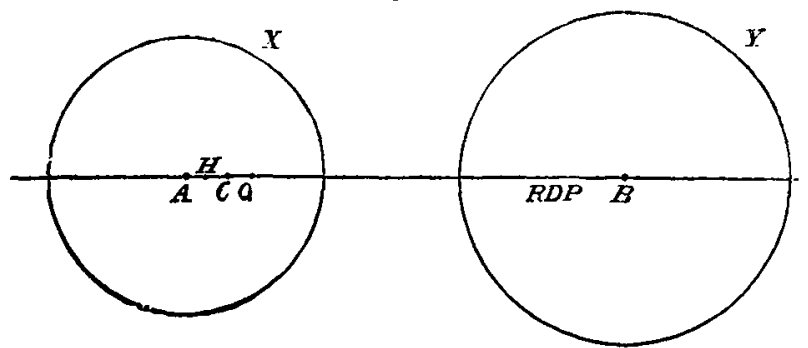

Let A, B be the centres of the spheres (fig. 3 ) ; $a, b$ the radii. Then $\dot{a}, \dot{b}$ are the normal velocities at the surfaces. 
Let $\phi_{A}$ be the velocity-potential in the liquid when $A$ pulsates and $B$ does not, $\phi_{B}$ the velocity-potential when $B$ pulsates and A does not. Then $\phi_{A}+\phi_{B}$ is the velocity-potential when both pulsate.

For if $n_{1}, n_{2}$ are normals to $\mathrm{X}, \mathrm{Y}$, drawn into the liquid,

$$
d \phi_{\mathrm{A}} / d n_{1}=\dot{a}, \quad d \phi_{\mathrm{B}} / d n_{2}=\dot{b}, \quad d \phi_{\mathbf{A}} / d n_{2}=0, \quad d \phi_{\mathrm{B}} / d n_{1}=0 .
$$

Therefore, if $\phi_{\mathrm{A}}+\phi_{\mathrm{B}}=\phi$,

$$
d \phi / d n_{1}=\dot{a} \text { and } d \phi / d n_{2}=\dot{b} .
$$

Also $\phi$ satisfies Laplace's equation, and vanishes at infinity.

If $\mathrm{T}$ be the kinetic energy of the liquid, $\rho$ its density,

$$
\begin{aligned}
\frac{2 \mathrm{~T}}{\rho} & =\iiint\left\{\left(\frac{d \phi}{d x}\right)^{2}+\left(\frac{d \phi}{d y}\right)^{2}+\left(\frac{d \phi}{d z}\right)^{2}\right\} d x d y d z \\
& =-\iint \phi \frac{d \phi}{d n_{1}} d \mathrm{~S}_{1}-\iint \phi \frac{d \phi}{d n_{2}} d \mathrm{~S}_{2}, . . .
\end{aligned}
$$

the volume-integration extending through the liquid, and the surface-integrals being taken over $S_{1}, S_{2}$, the surfaces of $X$ and $\mathrm{Y}$.

But by the surface conditions (1) and by Green's theorem,

$$
\iint \phi_{\mathrm{B}} \frac{d \phi}{d n_{1}} d \mathrm{~S}_{1}=\iint \phi_{\mathrm{A}} \frac{d \phi}{d n_{2}} d \mathrm{~S}_{2} . \ldots
$$

Substituting $\phi_{A}+\phi_{B}$ for $\phi$ in (2), and employing (1) and (3), we have

$$
2 \mathrm{~T} / \rho=-\dot{a} \iint \phi_{\mathrm{A}} d \mathrm{~S}_{1}-2 \dot{b} \iint \phi_{\mathrm{A}} d \mathrm{~S}_{2}-\dot{b} \iint \phi_{\mathrm{B}} d \mathrm{~S}_{2} .
$$

Let $\mathrm{P}$ (fig. 3) be the inverse of $\mathrm{A}$ in the sphere $\mathrm{Y} ; \mathrm{Q}, \mathrm{C}$ the inverses of $P, B$ in $X ; R, D$ the inverses of $Q, C$ in $Y$.

If $Y$ were absent, the pulsations of $X$ would give the same velocity-potential as a source $a^{2} \dot{a}$ at $\mathrm{A}$.

The image of this in $\mathrm{Y}$ is a line of doublets $\mathrm{BP}$; that of $\mathrm{BP}$ in $\mathrm{X}$ is a line of doublets $\mathrm{QC}$; that of $\mathrm{QC}$ in $\mathrm{Y}$ is a line of doublets RD; and so on.

Now the flow across the surface $\mathrm{X}$ due to $\mathrm{BP}, \mathrm{QC} ; \mathrm{RD}, \ldots$ taken in successive pairs is zero; and the flow across $Y$ due to $\mathrm{A}, \mathrm{BP} ; \mathrm{QC}, \mathrm{RD} ; \ldots$ taken in pairs is also zero.

$\phi_{A}$ is therefore the velocity-potential due to these systems.

It is simpler to transform each line of doublets into the equivalent source and line-sink.

Let $\mathrm{AB}=c$, and denote $a^{2} \dot{a}$ by $\mu$. 
The doublets BP reduce to a source $\mu b / c$ at $\mathrm{P}$ and a line$\operatorname{sink}-\frac{\mu b}{c . \overline{B P}}$ along $\mathrm{BP}$.

The doublets $\mathrm{QC}$ reduce to a source $\mu a b /\left(c^{2}-b^{2}\right)$ at $\mathrm{Q}$ and a line-sink $-\frac{\mu a b}{\mathrm{QC}\left(c^{2}-\dot{b}^{2}\right)}$ along QC.

We shall find that the doublets RD contribute a term of order $c^{-10}$ to $\mathrm{T}$; this we shall neglect.

The lengths of successive line-sinks diminish very rapidly, each being to its predecessor in a ratio of the order $a^{2} / c^{2}$ or $b^{2} / c^{2}$.

Therefore, since $\mathrm{BP}=b^{2} / c$, the lengths $\mathrm{QC}, \mathrm{RD}, \ldots$ are of order $c^{-3}, c^{-5} \ldots$, and the strengths of successive sources $\mathrm{A}, \mathrm{P}, \mathrm{Q}, \ldots$ are of order $1, c^{-1}, c^{-2} \ldots$

If a source $\nu$ at distance $r$ from $A$ contribute a term $\phi_{\nu}$ to $\phi_{A}$, it has been proved by Gauss that

$\iint \phi_{\nu} d \mathrm{~S}_{1}=-4 \pi a^{2} v / r$ or $-4 \pi a v$, according as $r>$ or $<a$.

[This may be proved in the following elementary way :-

Let the sphere be an attracting shell of surface-density unity.

$\iint \phi_{\nu} d \mathrm{~S}$ is the work done by a particle $\nu$ if the sphere recedes to infinity.

And $-4 \pi a^{2} v / r$ or $-4 \pi a \nu$ is the work done by the sphere when the particle recedes to infinity, according as $r>$ or $<a$.

Excepting the source $\mu$ at $A$, all the images within $A$ are of total strength zero.

An image outside A consists of a source of strength $s$ distant $\rho$ from $A$, and a line-sink - $s / \gamma$ per unit-length, with extremities distant $\rho, \rho+\gamma$ from A.

This contributes to the coefficient of $4 \pi a^{2}$ in $-\iint \phi_{A} d \mathrm{~S}_{1}$ the term

or

$$
\frac{s}{\rho}-\int_{0}^{\gamma} \frac{s}{\gamma} \frac{d x}{\rho+x}
$$

$$
\frac{s}{\rho}-\frac{s}{\gamma} \log _{e}\left(1+\frac{\gamma}{\rho}\right), \ldots . . . .
$$

which is, approximately,

For the image $\mathrm{PB}$,

$$
s \gamma / 2 \rho^{2}-s \gamma^{2} / 3 \rho^{3} . . . . . . . .
$$

$$
s=\mu b / c ; \gamma=b^{2} / c ; \rho=c-b^{2} / c . . .
$$


Therefore this contributes

$$
\frac{\mu b}{c^{2}-b^{2}}-\frac{\mu}{b} \log \frac{c^{2}}{c^{2}-b^{2}}
$$

The source at $\mathrm{R}$ is of order $c^{-3}$; $\mathrm{RD}$ is of order $c^{-5}$; $\mathrm{AR}$ is of order $c$.

Therefore RD contributes a term of order $c^{-10}$.

Omitting terms of this order,

$$
-\dot{a} \iint \phi_{\mathrm{A}} d \mathrm{~S}_{1}=4 \pi a^{4} \dot{a}^{2}\left\{\frac{1}{a}+\frac{b}{e^{2}-b^{2}}-\frac{1}{b} \log \frac{c^{2}}{c^{2}-b^{2}}\right\} ;
$$

$-\dot{b} \iint \phi_{\mathrm{B}} d \mathrm{~S}_{2}$ can be written down by interchanging $a$ and $b$.

It remains to form the term $-\iint \phi_{A} d S_{2}$ in (4).

A contributes $a^{2} \dot{a} / c$ to the coefficient of $4 \pi b^{2}$; BP contributes nothing.

For QC, $s=\mu a b /\left(c^{2}-b^{2}\right) ; \quad \rho=c-a^{2} c /\left(c^{2}-b^{2}\right), \quad \rho$ being here reckoned from $\mathbf{B}$;

$s \gamma^{2} / 3 \rho^{3}$ is of order $c^{-11}$;

$$
\gamma=\frac{a^{2} b^{2}}{c\left(c^{2}-b^{2}\right)} . \quad . \quad . \quad . \quad . .
$$

$$
\frac{s \gamma}{2 \rho^{2}}=\frac{\mu a^{3} b^{3}}{c^{7}}\left(\frac{1}{2}+\frac{a^{2}+b^{2}}{c^{2}}\right) ;
$$

RD contributes nothing, and further images are negligible.

Therefore, to terms of order $c^{-9}$ inclusive,

$$
\begin{aligned}
\frac{2 \mathrm{~T}}{\rho}=4 & \pi a^{4} \dot{a}^{2}\left\{\frac{1}{a}+\frac{b}{c^{2}-b^{2}}-\frac{1}{b} \log \frac{c^{2}}{c^{2}-b^{2}}\right\} \\
+ & 4 \pi b^{4} \dot{b}^{2}\left\{\frac{1}{b}+\frac{a}{c^{2}-a^{2}}-\frac{1}{a} \log \frac{c^{2}}{c^{2}-a^{2}}\right\} \\
+ & 8 \pi a^{2} b^{2} \dot{a} \dot{b}\left\{\frac{1}{c}+\frac{a^{3} b^{3}}{2 c^{7}}+\frac{a^{3} b^{3}\left(a^{2}+b^{2}\right)}{c^{9}}\right\}
\end{aligned}
$$

Now the force tending to increase $c$, required to maintain $c$ at its given value, is $-\frac{d \mathrm{\Gamma}}{d c}$.

Therefore, if the spheres are free to move they behave as if a force $\frac{d \mathrm{~T}}{d c}$ tended to separate them, and (omitting for brevity terms beyond $c^{-8}$ ),

$\frac{1}{\rho} \frac{d \mathrm{~T}}{d c}=-\frac{4 \pi a^{2} b^{2} \dot{a} b}{c^{2}}\left(1-\frac{7 a^{3} b^{3}}{2 c^{6}}\right)-\frac{4 \pi a^{4} \dot{a}^{2} b^{3}}{c^{5}}\left(1+\frac{2 b^{2}}{c^{2}}\right)-\frac{4 \pi b^{4} b^{2} a^{3}}{c^{5}}\left(1+\frac{2 a^{2}}{c^{2}}\right)$. 
If $\bar{a}, \bar{b}$ be the mean radii of the spheres, $\mathrm{T}$ the period of vibration, $\alpha, \beta$ the amplitude of vibration,

$$
a=\bar{a}+\alpha \sin \frac{2 \pi t}{\mathrm{~T}}, \quad b=\bar{b}+\beta \sin 2 \pi\left(\frac{t}{\mathrm{~T}}+\epsilon\right) ;
$$

and, supposing the pulsations small, the mean value of the force is (after replacing $\bar{a}, \bar{b}$ by $a, b$ )

$$
\begin{aligned}
-\frac{8 \pi^{3} a^{2} b^{2}}{\rho \mathrm{T}^{2}}\{ & \frac{\alpha \beta}{c^{2}}\left(1-\frac{7 a^{3} b^{3}}{2 c^{6}}\right) \cos \frac{2 \pi \epsilon}{\mathrm{T}} \\
& \left.+\frac{a b}{c^{5}}\left(a \alpha^{2}+b \beta^{2}\right)+\frac{2 a^{2} b^{2}}{c^{7}}\left(b \alpha^{2}+a \beta^{2}\right)\right\} .
\end{aligned}
$$

If the spheres are not very near, terms after that involving $c^{-2}$ can be neglected, and $c$ tends to diminish or increase according as $\cos \frac{2 \pi \epsilon}{\mathrm{T}}$ is positive or negative-i.e. according as the phases of vibration of the spheres approach more nearly to coincidence or opposition.

$\$ 6$. The above method is also applicable when the displacement on each sphere is a Zonal Harmonic with the line of centres as axis.

Definition.-If $(f, 0,0)(x, y, z)$ be the coordinates of two points $\mathrm{C}$ and $\mathrm{P}$, at a distance $r$ apart, a source at $\mathrm{C}$ which gives a potential $-\mu \frac{d^{n}}{d f^{n}}\left(\frac{1}{r}\right)$ at $\mathrm{P}$ is called a source of the
$n$th order.

A doublet is a source of the first order, and a source of the second order is derived from it in the same way as a doublet from a source of order zero (hitherto called a source). A source of the $r$ th order is derived similarly from a source of the $r-1$ th order.

Let $H$ (fig. 3) be the position of a doublet $\mu$ distant $f$ from $\mathrm{B}$ along $\mathrm{BA}$; and let $\mathrm{V}$ be the potential at $(x, y, z)$ due to $H$ and its successive images in $\mathrm{Y}$ and $\mathrm{X}$.

Take a doublet $\mu$ at $\mathrm{H}^{\prime}$ such that $\mathrm{BH}^{\prime}=f+d f$, and a doublet $-\mu$ at $\mathrm{H}$.

The potential at $(x, y, z)$ due to $\mathrm{H}^{\prime}$ and its images is $\mathrm{V}+\frac{d V}{d f} d f$.

Therefore the potential due to a source $\mu$ of the second order and its images is $\frac{d V}{d f}$, and that due to a source $\mu$ of the $n$th order and its images is $\frac{d^{n-1} V}{d f^{n-1}}$. 
When the axes of the doublet and of the derived sources are directed towards $\mathrm{B}$, this becomes $(-)^{n-1} \frac{d^{n-1} V}{d f^{n-1}}$.

Now let the spheres $X, Y$ vibrate so that the normal velocities are Zonal Harmonics $u \mathrm{P}_{n}, v \mathrm{P}_{m}$, having $\mathrm{AB}, \mathrm{BA}$ as their respective axes.

Then, since

$$
\mu \frac{d^{n}}{d f^{n}}\left(\frac{1}{r^{n}}\right)=\frac{\mu n ! \mathrm{P}_{n}}{r^{n+1}}
$$

$\mathrm{X}$, if it vibrated by itself, would behave like a source of the $n$th order of strength $\frac{a^{n+2} u}{n+1 !}$, with its axis along $\mathrm{AB}$.

Let $\phi_{A}$ be the potential due to this source and its images. This is found by calculating successive images of the complex source $\mathrm{H}$ in $\mathrm{Y}$ and $\mathrm{X}$, and making $f=c$ after all operations are completed.

If $Y$ vibrated by itself it would be equivalent to a source of the $m$ th order, $\frac{b^{m+2} v}{m+1 !}$; and with its images it would give a potential $\phi_{\mathrm{B}}$.

Then, as before, if both spheres vibrate,

$$
2 \mathrm{~T} / \rho=-u \iint \phi_{\mathrm{A}} \mathrm{P}_{n} d \mathrm{~S}_{1}-2 v \iint \phi_{\mathrm{A}} \mathrm{P}_{m} d \mathrm{~S}_{2}-v \iint \phi_{\mathrm{B}} \mathrm{P}_{m} d \mathrm{~S}_{2} \text {. }
$$

To evaluate $\iint \phi_{A} \mathrm{P}_{m} d \mathrm{~S}_{2}$, we proceed as follows:-

$$
\phi_{\mathrm{A}}=(-)^{n-1} \frac{d^{n-1} \psi}{d f_{s}^{n-1}},
$$

where $\psi$ is the potential due to a doublet $\frac{a^{n+2} u}{n+1}$ ! (with axis along $\mathrm{AB}$ ) distant $f$ from $\mathrm{B}$, and to the images of the doublet; the subscript $c$ denoting that after all operations are completed $e$ is to be written for $f$.

$$
\therefore \iint \phi_{\mathbf{\Lambda}} \mathrm{P}_{m} d \mathrm{~S}_{2}=(-)^{n-1} \frac{d^{n-1}}{d f_{c}^{n-1}} \iint \psi \mathrm{P}_{m} d \mathrm{~S}_{2} .
$$

Now, if $\nu$ be a source of order zero distant $\xi$ from B, $\phi_{\nu}$ its velocity-potential,

$$
\iint \mathrm{P}_{m} \phi_{\nu} d \mathrm{~S}_{2}=-\frac{4 \pi b^{m+2} \nu}{(2 m+1) \xi^{m+1}} \text { or }-\frac{4 \pi \xi^{m} \nu}{(2 m+1) l^{m-1}}
$$

according as $\xi>$ or $<b$. 
Whence, for a doublet $v$ at the same point, with axis along $\mathrm{AB}$,

$$
\iint P_{m} \psi_{\nu} d S_{2}=-\frac{4 \pi b^{m+2} \nu(m+1)}{(2 m+1) \xi^{m+2}} \text { or } \frac{4 \pi m \xi^{m-1} \nu}{(2 m+1) b^{m-1}},
$$

$\psi_{\nu}$ being the velocity-potential due to $v$.

But taking $\xi>b$, the image of the doublet $\nu$ is $-v b^{3} / \xi^{3}$, distant $b^{2} / \xi$ from $\mathrm{B}$.

The two doublets together give the term

and it remains to evaluate

$$
-4 \pi \nu \frac{b^{m+2}}{\xi^{m+2}}
$$

$$
(-)^{n} 4 \pi \Sigma \frac{d^{n-1}}{d f_{c}^{n-1}}\left(\frac{b^{m+2} v}{\xi^{m+2}}\right)
$$

where the summation extends to the doublet $\frac{a^{n+2} u}{n+1 !}$ (or $\mu$ ) and its images outside $Y$. For this doublet

$$
\nu=\mu ; \quad \xi=f \text {. }
$$

For $Q$, the next image outside $B$,

$$
\nu=\mu \frac{a^{3} b^{3}}{\left(c f-b^{2}\right)^{3}} ; \quad \xi=c-\frac{a^{2} f}{c f^{\prime}-b^{2}}
$$

The succeeding image introduces a term of order $c^{-12}$ compared with that due to $\mu$; we shall neglect this.

Making $f=c$ after all operations are over, we have

where

$$
\begin{aligned}
& -v \iint \mathrm{P}_{m} \phi_{\mathrm{A}} d \mathrm{~S}_{2}=\frac{4 \pi a^{n+2} b^{m+2} u v}{c^{m+n+1}}\left\{\frac{m+n !}{m+1 ! n+1 !}\right. \\
& \left.+\frac{a^{3} b^{3}}{2 c^{6}}\left(1+\frac{\overline{m+2} \cdot a^{2}+n+2 \cdot b^{2}}{c^{2}}+\frac{p}{c^{4}}\right)+\ldots\right\},
\end{aligned}
$$

$p=(m+2)(m+3) a^{4}+2(m+2)(n+2) a^{2} b^{2}+(n+2)(n+3) l^{4}$

It remains to find $\iint \phi_{A} \mathrm{P}_{n} d \mathrm{~S}_{1}$, from which $\iint \phi_{B} \mathrm{P}_{m} d \mathrm{~S}_{2}$ can be derived by symmetry.

The source $\mu$ at $A$ contributes a constant, which we need not write.

The images $\mathrm{P}$ and $\mathrm{Q}$ contribute a term found by making

$$
\nu=\mu b^{3} / f^{3}, \quad \xi=c-b^{2} / f .
$$

The image $\mathbf{R}$ and its successor contribute only a small term; here $\xi$ can be regarded as constant, since $f$ only occurs in a term of order $e^{-5}$; and $\nu$ is approximately $\mu a^{3} b^{6} c^{-6} f^{-3}$. 
On Transient Electric Current from Iron and Nickel. 123

As far as terms of the order $c^{-(2 n+10)}$ inclusive, $-u \iint \mathrm{P}_{n} \phi_{\mathrm{A}} d \mathrm{~S}_{\mathrm{i}}$ is made up of the term

$$
\begin{aligned}
\frac{4 \pi a^{2 n+4} b^{3} u^{2}}{c^{2 n+4}}\left\{\frac{1}{2 !}\right. & +\frac{(n+2)^{2}}{3 !} \frac{b^{2}}{c^{2}}+\frac{(n+2)^{2}(n+3)^{2}}{4 ! 2 !} \frac{b^{4}}{c^{4}} \\
& \left.+\frac{(n+2)^{2}(n+3)^{2}(n+4)^{2}}{5 ! 3 !} \frac{b^{6}}{c^{6}}+\ldots\right\},
\end{aligned}
$$

due to the images $\mathrm{P}$ and $\mathrm{Q}$, together with the term

$$
\frac{2 \pi a^{2 n+4} a^{3} b^{6} u^{2}}{c^{2 n+10}}
$$

due to the image $R$.

By interchanging $m$ and $n, u$ and $v, a$ and $b$, the remaining term on the right-hand side of $(10)$ can be found.

If the vibrating spheres consist of liquid of density $\rho^{\prime}$, the velocity-potential within $\mathrm{X}$ is $u \mathrm{P}_{n} r^{n} / n a^{n-1}$, and the kinetic energy of the sphere $\mathrm{X}$ is $\frac{2 \pi \rho^{\prime} a^{3} u^{2}}{n(2 n+1)}$. A similar expression holds for the other sphere.

When $c$ is constant the principal term in the expression of the stress between the spheres varies as $c^{-(m+n+2)}$ or as $c^{-(2 n+5)}$, or as $c^{-(2 m+5)}$.

If $u, v$ are simple harmonic functions of the time, the average stress between the spheres is to be found as before.

The case when $m=n=1$ has been discussed by Mr. Hicks (loc. cit.); for all other values of $m$ and $n$ the centres of the spheres are at rest.

X. On Transient Electric Current produced by suddenly twisting Magnetized Iron and Nickel Wires. By H. NAGAOrA, Rigakusi, of Imperial University, Tokyo*.

$$
\text { [Plate II.] }
$$

TN a recent number of Wiedemann's Annalen, Herr 1 Zehnder + communicated the result of his experiments on the transient current produced by twisting magnetized iron and nickel wires, in which he finds the direction of current in nickel to be opposite to that in iron. The same result had been already known to me since July 1888 . Being engaged in

* Communicated by Sir William Thomson.

† "Ueber Deformationsströme." Wiedemann's Annalen, Bd. xxxriii. p. 68. This number reached me on 14th October, 1889. 